SAHUARUS

Revista Electrónica de Matemáticas,

Departamento de Matemáticas, Universidad de Sonora,

Vol. 2, No. 1, abril, 2017, pp. 49-64.

\title{
Novedosa Propuesta para las Ternas Pitagóricas de la Tableta Plimpton 322
}

\author{
Raúl Pérez-Enríquez \\ Universidad de Sonora \\ Instituto de Investigaciones Estéticas, UNAM \\ e-mail: raul.perez@unison.mx
}

\begin{abstract}
Resumen
Recientemente, he propuesto un nuevo enfoque de análisis para una de las tabletas más asombrosas de la antigua Mesopotamia: la Tableta Plimpton 322. La nueva visión de esta tableta se basa en considerarla como una tabla de registro de sitios sumerios; apoyándose en la propuesta de que las ternas de la tableta representan triángulos de Pitágoras Diofantinos con su cateto vertical (h) igual a un múltiplo de 6. Los quince triángulos rectángulos de la misma parecen tener su origen en Larsa y su contenido se ha debatido abundantemente en el pasado; sin embargo, nuestra aproximación a ella supone que las ternas de Pitágoras serían triángulos gnomónicos y no solo un simple ejercicio de un escribano. En este artículo, discuto, utilizando un método de análisis propuesto por E. Ley koo, el cálculo para las ternas con base 6; y por medio de la retro-ingeniería, encuentro nuevas ternas que podrían haber formado parte de la tableta en una parte ahora perdida de la misma. La metodología sugiere una columna adicional para el cateto (h) mismo con una columna más para el nombre del sitio de una "mojonera" para delimitar fronteras. También, muestro el programa computacional con el que se hallaron al menos dos nuevos sitios: uno para el triángulo 175:288:377 para la latitud de Larsa, y la terna 611:1020:1189 correspondiente a la latitud de Ur, en Mesopotamia.
\end{abstract}

\section{Introducción}

La tableta de arcilla con el número 322 del catálogo de la colección de G.A. Plimpton de la Universidad de Columbia fue descubierta en un lugar desconocido del desierto de Iraq y representa la tableta matemática más conocida de la antigua Mesopotamia. En ella, se inscriben datos numéricos de tiempos tan remotos como 1800 a. C.; datos que los analistas han identificado como 15 triángulos rectángulos que permiten catalogarla como la tableta matemática más avanzada anterior a las matemáticas griegas [6]. Si bien se desconoce su lugar de origen exacto y su contenido ha sido interpretado, la razón por la cual fue elaborada y si faltan partes a la misma aún está a debate. Eleonore Robson describe la tableta como un ejercicio de ternas de Pitágoras realizado por algún escribano y supone que Larsa, una antigua ciudad sumeria cerca de Uruk, pudo ser el lugar donde fue escrita [11]. En un trabajo más reciente, Britton y colaboradores hacen una descripción detallada de la tableta, lo que sugiere la posibilidad de una superficie de escritura ampliada más allá de los $125 \mathrm{~mm}$ por 83 mm en el anverso [1].

Larsa fue una ciudad importante de la antigua Mesopotamia. Su desarrollo viene de la época sumeria y podría considerarse contemporánea de otras ciudades como Ur, Uruk, Lagash y Nippur. Todas ellas ciudades que cultivaron la escritura cuneiforme y donde se han 
encontrado textos de muy diversas clases. La presencia de la Tableta Plimpton 322 en esta región permite reconocer a esta zona de Mesopotamia como uno de los sitios de desarrollo matemático más temprano como lo hace ver Neugebauer [6].

Hace un par de años, propuse reconocer en el triángulo gnomónico, gnomon y la sombra como los lados y el rayo de sol como la hipotenusa [7], [8], una herramienta para la definición de un concepto denominado factor gnomónico $(f g)$; estableciendo de esta manera, una conexión entre el tiempo (movimiento del sol a lo largo del día y a lo largo del año) y las matemáticas. Algunas ciudades antiguas importantes fueron situadas donde el factor gnomónico era un número entero: Teotihuacan y Chichén-Itzá en Mesoamérica con $f g=1$; Stonehenge en Reino Unido con un factor de 3; y Newgrange en Irlanda hace 5000 años, con $f g=4$ [8]; hecho que resulta significativo pues la manera de calcular el factor gnomónico (el cociente de la diferencia de sombras de un gnomon en los solsticios y la longitud del gnomon mismo) implica un conocimiento matemático implícito.

En este artículo describo, brevemente, la Tableta Plimpton 322, incluyendo la más reciente interpretación de Britton et al. [1] y el enfoque matemático aplicado por mí [9]. En seguida, discuto en detalle las ternas utilizando la metodología proporcionada por Dr Ley Koo para descubrir las familias de ternas de Pitágoras Diofantinas y su factor común: seis. Finalmente, propongo el uso de esta metodología para ubicar nuevos sitios probables que mejoran la interpretación de la Tableta.

\section{$2 \quad$ El Contenido de la Tableta}

Existen varias descripciones de la Tableta Plimpton 322 y ellas se pueden encontrar fácilmente en la literatura. O. Neugebauer fue quien por primera vez describió y dió una interpretación coherente de los datos numéricos de sus cuatro columnas [6]. La Figura 1 muestra una de las varias imágenes de esta importante tableta de arcilla. Un análisis y traducción de los textos cuneiformes indica que son quince triángulos cuyos lados son enteros; además, hay una fracción que representa un cociente al cuadrado entre los lados del triángulo; esto es, inscritos en las columnas dos y tres de la tableta proporcionan el ancho $(w)$ y la diagonal $(d)$; mientras que la primera columna muestra el cuadrado de la relación entre $d$ y el alto $(h)$ (ver Figura 3). La cuarta columna es solamente para establecer un número de orden (del 1 al 15).

Para facilitar la discusión en este trabajo, debo suponer que los triángulos de Pitágoras son Difantinos y que sus lados son los siguientes: anchura : altura $:$ diagonal $(w: h: d)$ con los correspondientes ángulos de $W: H: D$ como se muestra en la Figura 2; se considera a $D$ como ángulo de 90 grados. Además, estoy tomando los nombres de las columnas en la tableta y el lado de altura será evidente más adelante cuando éstos sean considerados triángulos gnomónicos.

Según Joyce, el contenido de la tableta es el que se muestra en la Figura 4. La primera de las columnas es $(d / h)^{2}$ [3]. La traducción de la primera cabeza de la columna puede leerse, según Robson, como "El lugar takiltum de la diagonal de la cual 1 es arrancado, para que salga el lado más corto" [11]. Los datos contenidos, en notación sexagecimal, son las de los 


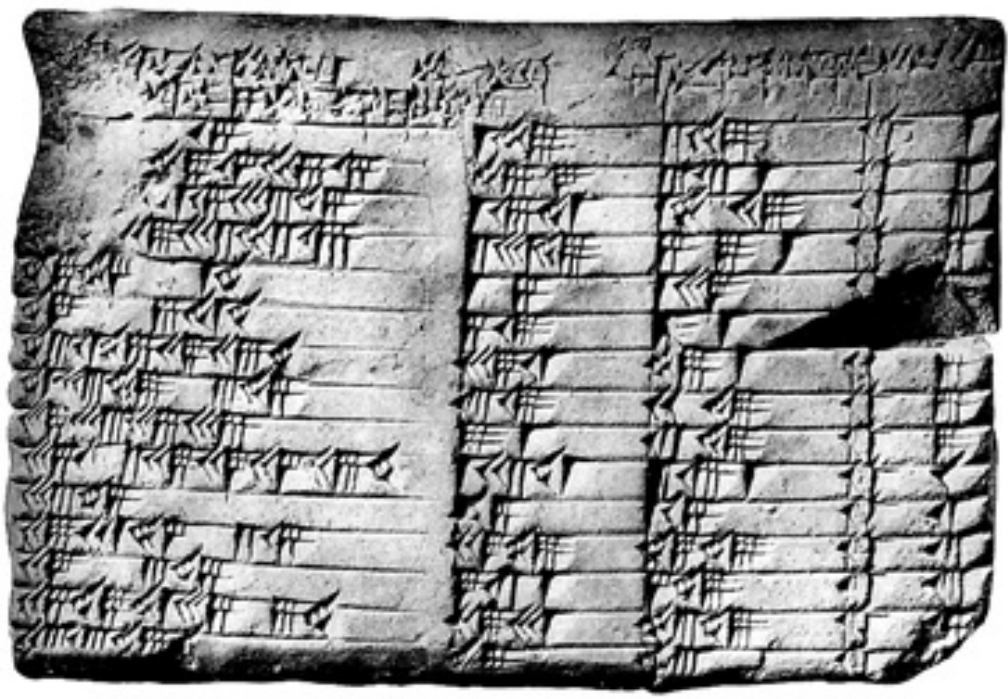

Figura 1: Tableta con número 322 del Catálogo de G.A. Plimpton Collection de la Universidad de Columbia, EUA [6].

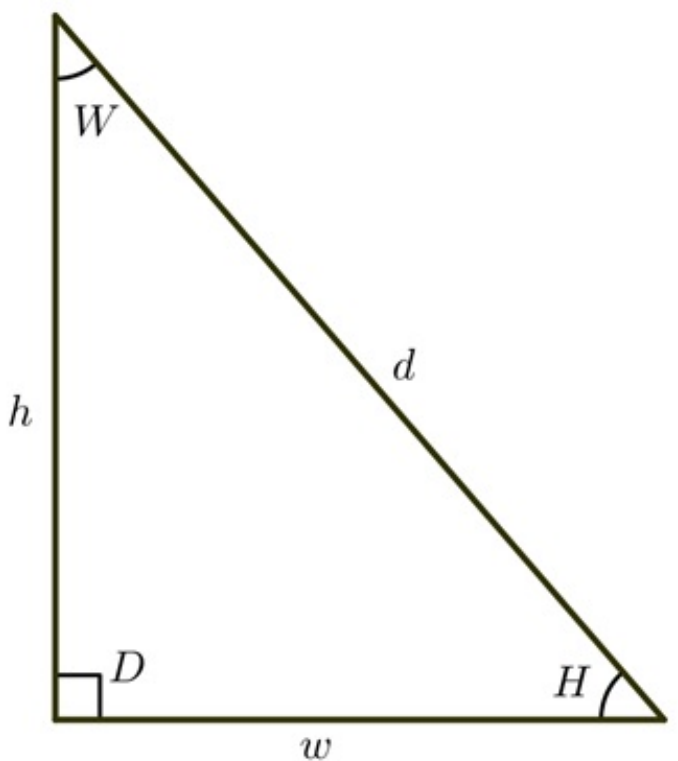

Figura 2: Descripción de los triángulos: anchura, altura y diagonal $(w: h: d)$ con los correspondientes ángulos de $W: H: D$.

triángulos que aparecen en la Figura 3.

Es relevante para esta discusión el tener en cuenta la propuesta hecha por Joyce considerando una columna faltante cuyo ángulo $W$ (él lo designa como ángulo A y estaría a la izquierda de la columna $1\left((d / h)^{2}\right.$ que él menciona como $\left.(c / b)^{2}\right)$ como se muestra en la Figura 4.

El nuevo enfoque que propongo para la interpretación de la Tableta es complementario 


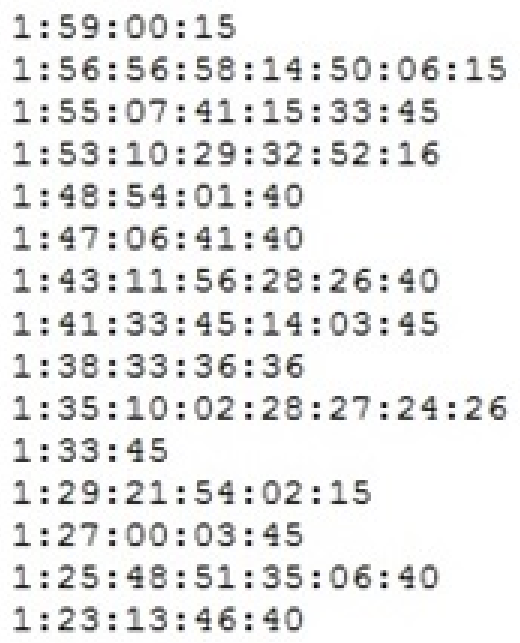

$\begin{array}{ccc}\text { width } & \text { diagonal } & \\ 1: 59 & 2: 49 & 1 \\ 56: 07 & 1: 20: 25 & 2 \\ 1: 16: 41 & 1: 50: 49 & 3 \\ 3: 31: 49 & 5: 09: 01 & 4 \\ 1: 05 & 1: 37 & 5 \\ 5: 19 & 8: 01 & 6 \\ 38: 11 & 59: 01 & 7 \\ 13: 19 & 20: 49 & 8 \\ 8: 01 & 12: 49 & 9 \\ 1: 22: 41 & 2: 16: 01 & 10 \\ 45 & 1: 15 & 11 \\ 27: 59 & 48: 49 & 12 \\ 2: 41 & 4: 49 & 13 \\ 29: 31 & 53: 49 & 14 \\ 56 & 1: 46 & 15\end{array}$

Figura 3: Columnas de la imagen de la Tableta Plimpton 322 con encabezado identificado; el encabezado de la primera columna contiene la palabra 'diagonal' en él y ha sido traducido [11]; los números están en notación sexagesimal.

\begin{tabular}{|c|c|}
\hline ng. & $(c / b)^{2}$ \\
\hline 44.76 & $1: 59: 00: 15$ \\
\hline 44.25 & $1: 56: 56: 58: 14: 50: 06: 15$ \\
\hline 43.79 & $1: 55: 07: 41: 15: 33: 45$ \\
\hline 43.27 & $1: 53: 10: 29: 32: 52: 16$ \\
\hline 42.08 & $1: 48: 54: 01: 40$ \\
\hline 41.54 & $1: 47: 06: 41: 40$ \\
\hline 40.32 & $1: 43: 11: 56: 28: 26: 40$ \\
\hline 39.77 & $1: 41: 33: 45: 14: 03: 45$ \\
\hline 38.72 & $1: 38: 33: 36: 36$ \\
\hline 37.44 & $1: 35: 10: 02: 28: 27: 24: 26$ \\
\hline 36.87 & $1: 33: 45$ \\
\hline 35.78 & $1: 31: 09: 09: 25: 42: 02: 15$ \\
\hline 34.98 & $1: 29: 21: 54: 02: 15$ \\
\hline 33.86 & $1: 27: 00: 03: 45$ \\
\hline 33.26 & $1: 25: 48: 51: 35: 06: 40$ \\
\hline 31.89 & $1: 23: 13: 46: 40$ \\
\hline
\end{tabular}

Figura 4: Imagen de tabla de Joyce con el ángulo propuesto A que corresponde al ángulo $W$ de este artículo [3].

a éste, tal y como fue publicado recientemente [9]. En este nuevo acercamiento, se considera que los triángulos de la Tableta podrían representar un conjunto de ternas Pitagóricas especialmente seleccionadas, obedeciendo una regla para ángulos $\mathrm{W}$ cuyos valores van de $45^{\circ}$ hasta $31^{\circ}$ y que pudieron estar relacionadas con las sombras de un gnomon de acuerdo con lo que se describe en la siguiente sección. 


\section{Factor Gnomónico y Latitudes de Sitios}

Un análisis del comportamiento de los triángulos descritos en la Tableta Plimpton 322 me dieron una idea acerca de cómo fueron seleccionados. ¿Por qué corresponden a un rango concreto de triángulos? ¿El hecho de que los valores de los ángulos sean casi equidistantes, en un intervalo que va de $32^{\circ}$ a $45^{\circ}$, tiene que ver con algún problema práctico?

Efectivamente, si se tienen en cuenta los valores de los ángulos $W$, mostrados en la Figura 4, como una representación de una latitud de algún sitio, se encontrará que todos ellos están en el intervalo de la antigua Mesopotamia: regiones sumerias y asirias, hasta la moderna Armenia y el Cáucaso. Y después de combinarlos con el ancho (w) como una sombra, el lado que falta sería la altura de un gnomon $(h)$. Entonces, las ternas que aparecen en la tableta comentada, serían, posiblemente, representaciones de sitios en donde, en los equinoccios, el sol de mediodía forma estos triángulos "Pitagóricos" [9].

Por otra parte, siguiendo el mapa de Mesopotamia y Asiria resulta fácil buscar algunos otros lugares importantes de la región: Babilonia, Larsa, Lagash, Ur. Sitios que uno podría esperar ver incluidos entre las ternas. Babilonia puede estar fuera del alcance pues no era tan importante como Larsa o Lagash hace 4000 años pero podría ubicarse entre las filas 14 y 15. La inclusión de Ur llevaría a abrir el intervalo hasta los $31^{\circ}$. Las ternas Pitagóricas Diofantinas de la Tableta Plimpton 322 completarían un cuadro no evidente en un principio.

Basado en la sugerencia hecha por L. Cottrell en el sentido de que los reyes Mesopotámicos hicieron uso de marcadores de piedra (mojoneras) para indicar las fronteras entre ciudades [2], he presentado una metodología que permite identificar un elemento común para todas las ternas de la mencionada tableta. Con los resultados obtenidos mediante el enfoque matemático que desarrollo más adelante, he logrado definir la posible ubicación de estos marcadores: con la ayuda de sus latitudes obtuvimos dos parámetros llamados $l$ y $k$ que fueron usados para calcular $h$ (ver Figura 2). Así, se confirma que las ternas Pitagóricas de la Tableta Plimpton 322 serían un registro de triángulos cuyo cateto $h$ es un múltiplo de seis.

Asimismo, esta metodología dio nuevas ternas, visibles en la Tabla 1, cuya ubicación estaría en Mesopotamia. Sin duda, Babilonia tuvo que ser incluido porque hay una brecha muy grande entre las latitudes adyacentes $\left(33,26^{\circ}\right.$ y $\left.31,89^{\circ}\right)$. Para el caso de Lagash no pude encontrar ninguna terna. La ciudad de Ur confirma su presencia a través del triángulo 611:1020:1189 en la latitud de $31^{\circ}$.

Tabla 1: Propuestas de filas faltantes la Tableta Plimpton 322

\begin{tabular}{lllllll}
\hline w & d & fila & h & H & W & Ciudad \\
\hline 1235 & 2293 & 14 bis & 1932 & 57.412 & 32.588 & Babilonia \\
175 & 337 & 16 & 288 & 58.716 & 31.284 & Larsa \\
611 & 1189 & 17 & 1020 & 59.078 & 30.922 & Ur \\
\hline
\end{tabular}


El caso de Larsa, lugar donde la fabricación de la tableta se realizó (según Robson), debe ser comentado por separado. De los cálculos hechos son varias las ternas que podrían corresponder a esa ciudad, sin embargo, la que aparece en la tabla (terna 175:288:337) sería la mejor situada. En la siguiente sección, explico la manera en que se llevaron a cabo los análisis de las ternas, mediante el método sugerido por Ley Koo, y el programa de cálculo con que se realizaron las iteraciones.

\section{Aproximación Analítica a las Ternas}

Deseo aclarar que la metodología descrita en esta sección no pretende ser una representación de los cálculos realizados por la gente de la antigua Mesopotamia, sino una forma en que se pueden reconstruir los datos de la tableta. El objetivo principal de este método es establecer una relación entre los ángulos $W$ y el cateto $h$. Una expresión general para las ternas Pitagóricas puede ser encontrada, por ejemplo, en el libro de Stillwell sobre historia de las matemáticas [12]; esta es:

$$
\begin{gathered}
d=\left(p^{2}-q^{2}\right) r, \\
h=2 p q r, \\
w=\left(p^{2}+q^{2}\right) r,
\end{gathered}
$$

donde $p, q$ y $r$ son números enteros. Sin embargo, para la deducción que aquí se propone se considerarán otras premisas.

Supóngase que todas las ternas de la tableta tienen el cateto $h$ igual a un múltiplo de 6 (realmente la mayoría de ellos son múltiplos de 30), entonces, los otros lados del triángulo deben ser impares; esto es, debe existir un número impar entre $d$ y $h$. Con esta hipótesis y suponiendo que $w$ es también un impar, me propongo encontrar los factores necesarios para relacionar el ángulo $W$ con el cateto $h$ como sigue:

Definición 4.1 Sea la estructura de los tringulos de la Tableta Plimpton 322 como sigue:

$$
\begin{gathered}
d=(6 n+2 k+1) \\
h=6 n ; \\
w=(2 m+2 k+1) .
\end{gathered}
$$

donde $n$ y $m$ son números enteros arbitrarios a ser determinados; $k$ es un factor entero complementario que permite hacer a d y $w$ números impares.

Teorema 4.2 Los enteros $n$ y $m$ están relacionados de la siguiente manera:

$$
n=\frac{1}{3}\left(\frac{m^{2}}{2 k+1}+m\right) .
$$

Demostración. Como una terna Pitagórica está definida por la condición

$$
d^{2}-h^{2}=w^{2}
$$

entonces, 


$$
(d-h)(d+h)=w^{2}
$$

por lo tanto,

$$
(6 n+2 k+1-6 n)(12 n+2 k+1)=(2 m+2 k+1)^{2}
$$

o bien,

$$
12 n(2 k+1)+(2 k+1)^{2}=4 m^{2}+4 m(2 k+1)+(2 k+1)^{2}
$$

Lo cual puede ser simplificado a

$$
12 n=\frac{4 m^{2}}{2 k+1}+4 m
$$

$y$, por tanto,

$$
n=\frac{1}{3}\left(\frac{m^{2}}{2 k+1}+m\right)
$$

q.e.d.

Lema 4.3 Una vez demostrado el Teorema, se puede hacer una siguiente suposición: $m$ es también un múltiplo de $(2 k+1)$; por ejemplo,

$$
m=3 l(2 k+1)
$$

con l como un número real. Entonces, la expresión para $n$ es

$$
n=\left(3 l^{2}+l\right)(2 k+1)
$$

Con ayuda de esta expresión la terna de Plimpton 322 queda como

$$
\begin{gathered}
d=6\left(3 l^{2}+l\right)(2 k+1)+(2 k+1) \\
h=6\left(3 l^{2}+l\right)(2 k+1) \\
w=6 l(2 k+1)+(2 k+1)
\end{gathered}
$$

o bien,

$$
\begin{gathered}
d=6\left(3 l^{2}+l+\frac{1}{6}\right)(2 k+1) ; \\
h=6\left(3 l^{2}+l\right)(2 k+1) ; \\
w=6\left(l+\frac{1}{6}\right)(2 k+1) .
\end{gathered}
$$


Ahora se puede recordar que mi interés está orientado a obtener las ternas para ciertos valores de la latitud; esto es, para ciertos valores de $W$. Por ello es posible introducir un nuevo teorema.

Teorema 4.4 Sea $x=\tan W$. El valor de l se puede hallar como solución de la siguiente ecuación cuadrática

$$
3 x l^{2}+(x-1) l-\frac{1}{6}=0 .
$$

Demostracin. Si $x$ es el valor de la tangente de $W$ entonces

$$
x=w / h
$$

y resolviendo con las expresiones (7) y (8) se tiene

$$
\begin{gathered}
x=\tan W=\frac{6\left(l+\frac{1}{6}\right)(2 k+1)}{6\left(3 l^{2}+l\right)(2 k+1)} \\
3 x l^{2}+l x=l+\frac{1}{6}
\end{gathered}
$$

o bien,

$$
3 x l^{2}+(x-1) l-\frac{1}{6}=0
$$

cuya solución es

$$
l=\frac{-(x-1)+\sqrt{(x-1)^{2}+2 x}}{6 x}
$$

q.e.d.

Con ayuda de los valores calculados de $l$ y las alturas $h$ de $(7)$ es posible obtener los valores correspondientes de $k$. Para ello, se debe considerar

$$
2 k+1=\frac{h}{6\left(3 l^{2}+l\right)}
$$

o bien,

$$
k=\frac{h}{12\left(3 l^{2}+l\right)}-\frac{1}{2}
$$

Así, suponiendo que los valores de $W$ y $H$ están dados en las hileras de la Tableta Plimpton 322, los valores de $l$ y $k$ pueden ser calculados. En la Tabla 2, se muestran los ángulos $H$ y $W$ para cada una de las ternas de la Tableta. Asimismo, aparecen en ella las alturas $h$ calculadas utilizando (7) a partir de los valores de $l$ y $k$, obtenidos con las expresiones (10) y (11).

Puede entenderse que las alturas que se muestran en la Tabla 2 pueden obtenerse de los valores conocidos de las ternas de la tableta Plimpton 322. En consecuencia, las ternas que aparecen en la Tabla 1 han sido obtenidas por el uso de una retro-ingeniería por iteración tomando como punto de partida las latitudes de las ciudades Mesopotámicas de Babilonia, Larsa y Ur. 
Tabla 2: Valores de $l$ y $k$ para ternas de Plimpton 322

\begin{tabular}{lllllll}
\hline Num. & $h$ & $H$ & $W$ & $x$ & $l$ & $k$ \\
\hline 1 & 120 & 45.240 & 44.760 & 0.992 & 0.238 & 25 \\
2 & 3456 & 45.747 & 44.253 & 0.974 & 0.243 & 685 \\
3 & 4800 & 46.213 & 43.787 & 0.959 & 0.248 & 925 \\
4 & 13500 & 46.729 & 43.271 & 0.941 & 0.254 & 2521 \\
5 & 72 & 47.925 & 42.075 & 0.903 & 0.267 & 13 \\
6 & 360 & 48.455 & 41.545 & 0.886 & 0.273 & 61 \\
7 & 2700 & 49.685 & 40.315 & 0.849 & 0.287 & 421 \\
8 & 960 & 50.230 & 39.770 & 0.832 & 0.294 & 145 \\
9 & 600 & 51.282 & 38.718 & 0.802 & 0.308 & 85 \\
10 & 6480 & 52.563 & 37.437 & 0.766 & 0.325 & 841 \\
11 & 60 & 53.130 & 36.870 & 0.750 & 0.333 & 8 \\
12 & 2400 & 55.024 & 34.976 & 0.700 & 0.362 & 265 \\
13 & 240 & 56.145 & 33.855 & 0.671 & 0.381 & 25 \\
14 & 2700 & 56.738 & 33.262 & 0.656 & 0.391 & 265 \\
15 & 90 & 58.109 & 31.891 & 0.622 & 0.417 & 85 \\
\hline
\end{tabular}

\section{Latitudes Perdidas: el programa}

En la Figura 5, se presenta el pesudocódigo de un programa en lenguaje 'C' que permite encontrar las Ternas Pitagóricas obedeciendo las reglas expuestas en la sección anterior, en donde la información de entrada es la latitud deseada (ver el listado en el Apéndice). La estructura del programa es directa y para la latitud proporcionada encuentra las ternas de Pitágoras correspondientes. En los ejemplos que aparecen en la Figura 6, se considera el caso de Larsa.

\section{Comentarios Finales}

A lo largo de este trabajo se ha presentado una metodología para el análisis de la Tableta Plimpton 322. La metodología toma en consideración el hecho de que la tableta permite calcular el ángulo $W$ de los triángulos inscritos y su complementario $H$. Encontrando, a partir de este dato, el cateto $h$ bajo la hipótesis de que dicho valor es múltiplo de seis (6), y de que los lados $w$ y $d$ están separados de él por números impares; además, se supone que los ángulos $W$ están entre $32^{\circ}$ y $45^{\circ}$.

Esta metodología va ligada con la interpretación de que un triángulo rectángulo puede ser una representación de un gnomon $(h)$ y su sombra $(w)$. De manera que los ángulos $W$ se interpretan como la latitud de un sitio donde, en los equinoccios, dicho triángulo rectángulo fue observado. Con ello, se ha propuesto que las ternas Pitagóricas de Tableta Plimpton 322 podrían haber definido lugares ubicados a lo largo de una posible ruta entre regiones de 


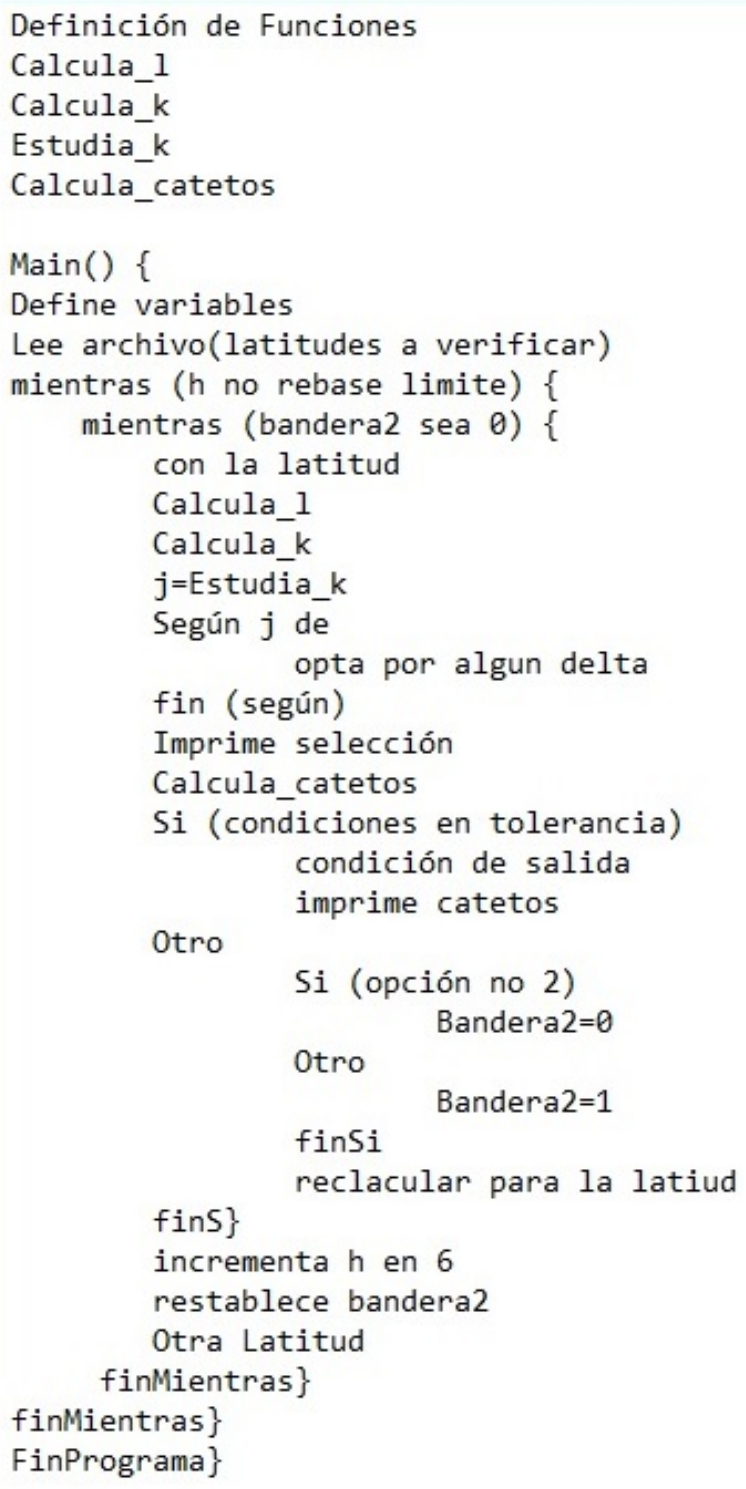

Figura 5: Pseudocódigo del Programa Plimpton322.c (ver código en el Apéndice).

Mesopotamia en la época sumeria.

Se reporta, asimismo, que se han hallado ternas para latitudes de Babilonia, Larsa y Ur que bien podrían estar en la Tableta Plimpton 322 en las filas 14 bis, 16 y 17. Si se recuerda que la tableta fue recuperada quebrada, se podría sugerir la siguiente hipótesis: Babilonoia fue el sitio en donde un escribano la elaboró y, al menos, le faltan dos filas como otros autores han afirmado.

De esta manera, la tableta Plimpton 322 podría interpretarse con una nueva perspectiva: Una tabla de los lugares donde se colocaron "marcadores de piedra" para delimitar regiones [9]. La Figura 7 muestra cómo se distribuyen las ternas o triángulos de la Tableta cuando se utiliza un gnomon de una unidad. 

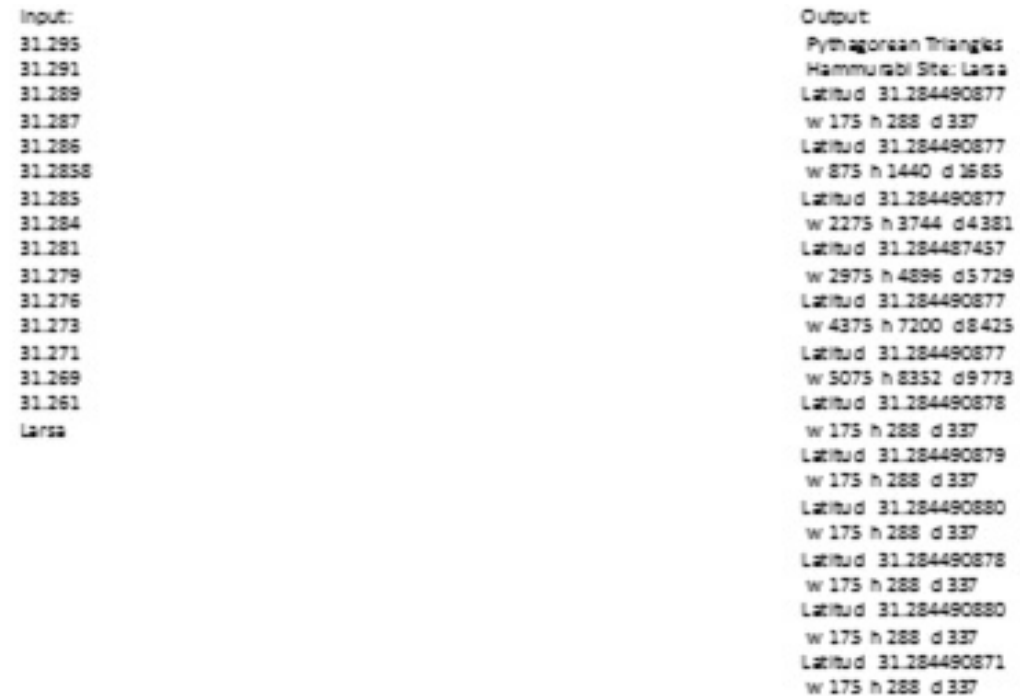

Figura 6: Imágenes de los datos de entrada (izquierda) y de salida (derecha) para el caso de Larsa.

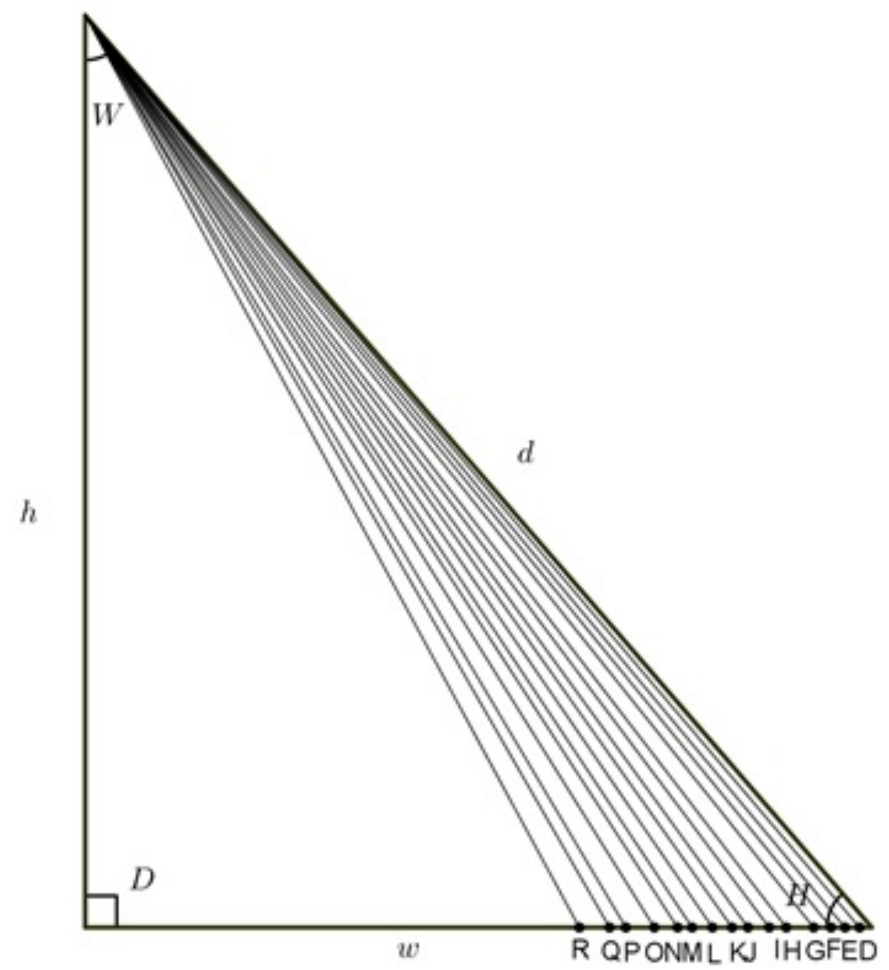

Figura 7: Los Triángulos de la Tableta Plimpton de 322 formados por un gnomon común de una unidad de altura y sombras identificadas de $\mathrm{D}$ a $\mathrm{R}$ debido a la elevación del Sol en el equinoccio.

Un último tema que se puede abordar aquí es: ¿por qué los datos de la Tableta comienzan en $32^{\circ}$ y no cubre un intervalo de 30 a 45 grados? Desde un punto de vista matemático, 
sería 30 grados el ángulo más lógico para acabar la tabla, si la interpretación de la Tableta como ejercicio de un escribano [11] fuera tomada como válida. Sin embargo, en la propuesta que presento, la latitud de $31^{\circ}$ parece más factible debido a que el Golfo Pérsico en tiempos sumerios, con la ciudad de Eridu, alcanzaría un poco más de ese límite.

\section{Agradecimientos}

Deseo agradecer al Dr Eugenio Ley Koo sus aportaciones a este trabajo y su revisión del texto. Deseo reconocer a la UNISON por el permiso sabático y a la UNAM, al Instituto de Investigaciones Estéticas, por haberme acogido durante mi estancia sabática.

\section{Referencias}

[1] Britton, J.P., Proust, C., Shnider, S., Plimpton 322: a review and a different perspective, Arch. Hist. Exact Sci., 65, 65, 519566 (2011)

[2] Cottrell L., Land of the Two Rivers, The World Publishing Col, Cleveland (1962)

[3] http://aleph0.clarku.edu/-djoyce/mathhist/plimpnote.html

[4] http://GoogleEarth.com

[5] Montané Martí, J.C. ,Fundamentos para una Teoría Arqueológica, 18,INAH, Hermosillo (1980)

[6] Neugebauer O., The Exact Sciences in Antiquity, pp. 37 - 39, Dover, New York (1969)

[7] Perez-Enriquez R., Trilithons and a three-season Solar Calendar at Stonehenge(2000)

[8] Perez-Enriquez, R., Platos Triangle And Gnomonic Factor: An Application To Herodotus Oracles, Mediterranean Archaeology and Archaeometry, 14, 3, 45 - 53 (2014)

[9] Perez-Enriquez, R., Plimpton 322 Tablet as a Sumerians Ancient Boundaries Record, ELECTRYONE, 3, 2, 20 -33 (2015)

[10] Robson, E., Neither Sherlock Holmes nor Babylon: A reassessment of Plimpton 322, Historia Mathematica, 28, 3, 167- 206 (2001)

[11] Robson, E., Words and Pictures: New Light on Plimpton 322, The American Mathematical Monthly, 109, , 105 - 120 (2002)

[12] Stillwell, J.,Mathematics and Its History, pp. 4 - 6, Springer, New York (2010) 


\section{Apéndice}

Listado del Programa Plimpton.c:

Programa Plimpton_v4.c

\#include <stdio.h $>$

\#include <math.h>

\#include <stddef .h>

\#include <stdlib.h>

\#include <locale.h>

/* program \$Pythagorean\_triplet $\$$

Version $4 * 1$

float \$calcula \_l (double X ) \{

float $f, L$;

$f=X-1.0$;

$L=(-f+\operatorname{sqrt}(f * f+2.0 * X)) /(6 * X)$;

printf("L $\backslash \%$ f $X \% f \quad \backslash n ", L, X) ; * /$

return (L); \\

\}

float calcula \_k(int $\mathrm{H}$, float $\mathrm{L}) \quad\{$

float $f, K$;

$\mathrm{f}=12.0 *(3 * \mathrm{~L} * \mathrm{~L}+\mathrm{L})$;

$\mathrm{K}=($ float $) \mathrm{H} / \mathrm{f}+0.5$;

return $(\mathrm{K})$;

\}

int Estudia $\backslash$ k (float $K) \quad\{$

int $\mathrm{J}=2, \mathrm{P}, \mathrm{M}$;

$\mathrm{P}=$ (int) $\mathrm{K}$;

$\mathrm{M}=\mathrm{P} \quad \backslash \% 2$;

if $(\mathrm{M}==0)$

if $(\mathrm{K}-\mathrm{P}>0.97) \quad \mathrm{J}=0$;

/* else if $(\mathrm{K}-\mathrm{P}<0.02) \quad \mathrm{J}=3$;

else $\mathrm{J}=2 ; * /$

else if $(\mathrm{K}-\mathrm{P}<0.03) \mathrm{J}=1$;

/* else if $(\mathrm{K}-\mathrm{P}>0.98) \quad \mathrm{J}=4 ; * /$

else $\mathrm{J}=2$;

/* $\quad$ printf(" $\mathrm{K} \backslash \%$ f $\mathrm{P} \backslash \%$ d $\mathrm{M} \backslash \% \mathrm{~d} \backslash \mathrm{n}$ ", $\mathrm{K}, \mathrm{P}, \mathrm{M}) ; * /$

return $(\mathrm{J})$;

\}

int calcula \_catetos(float L,float $K$,float* H,float* D,float* W) \{ 


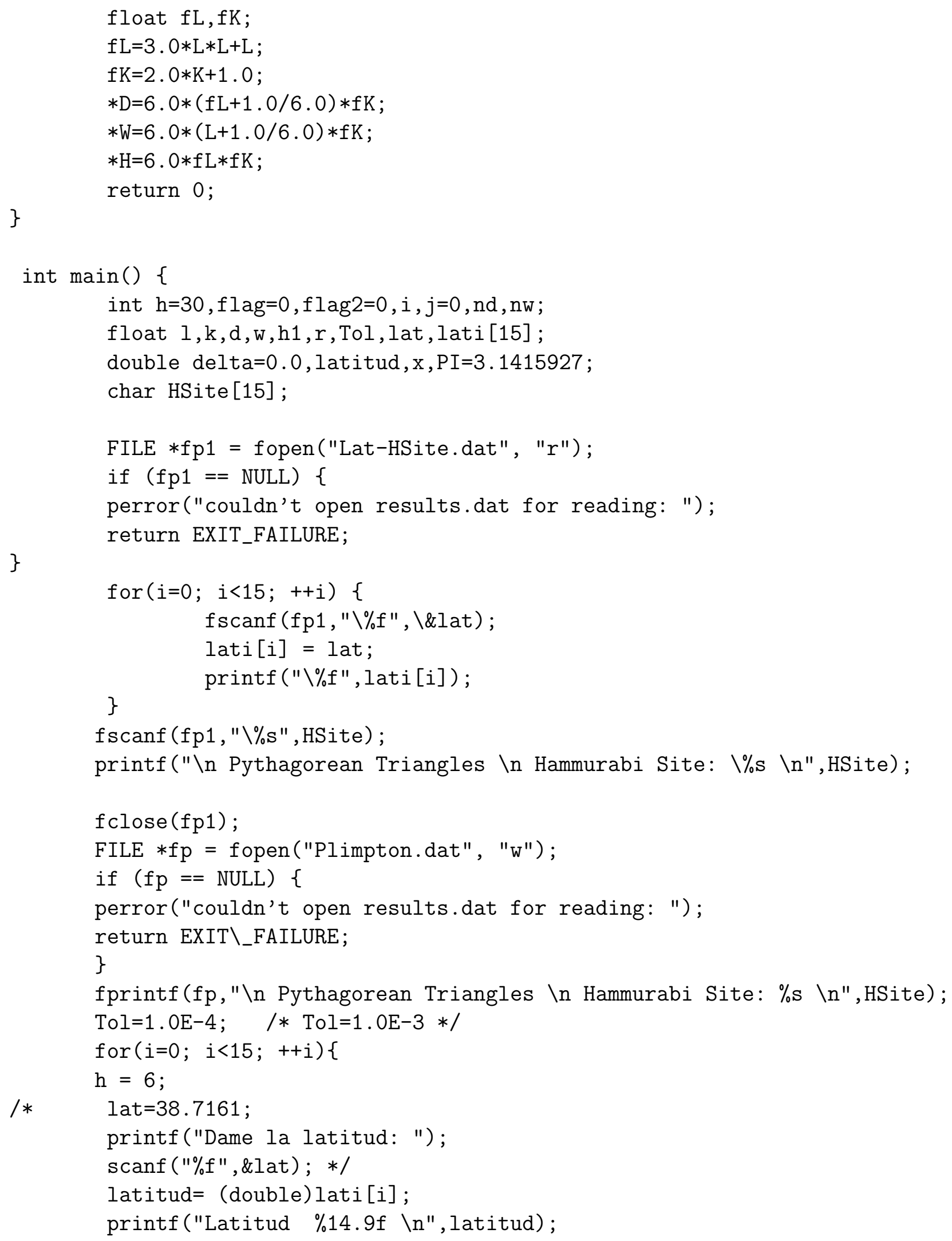




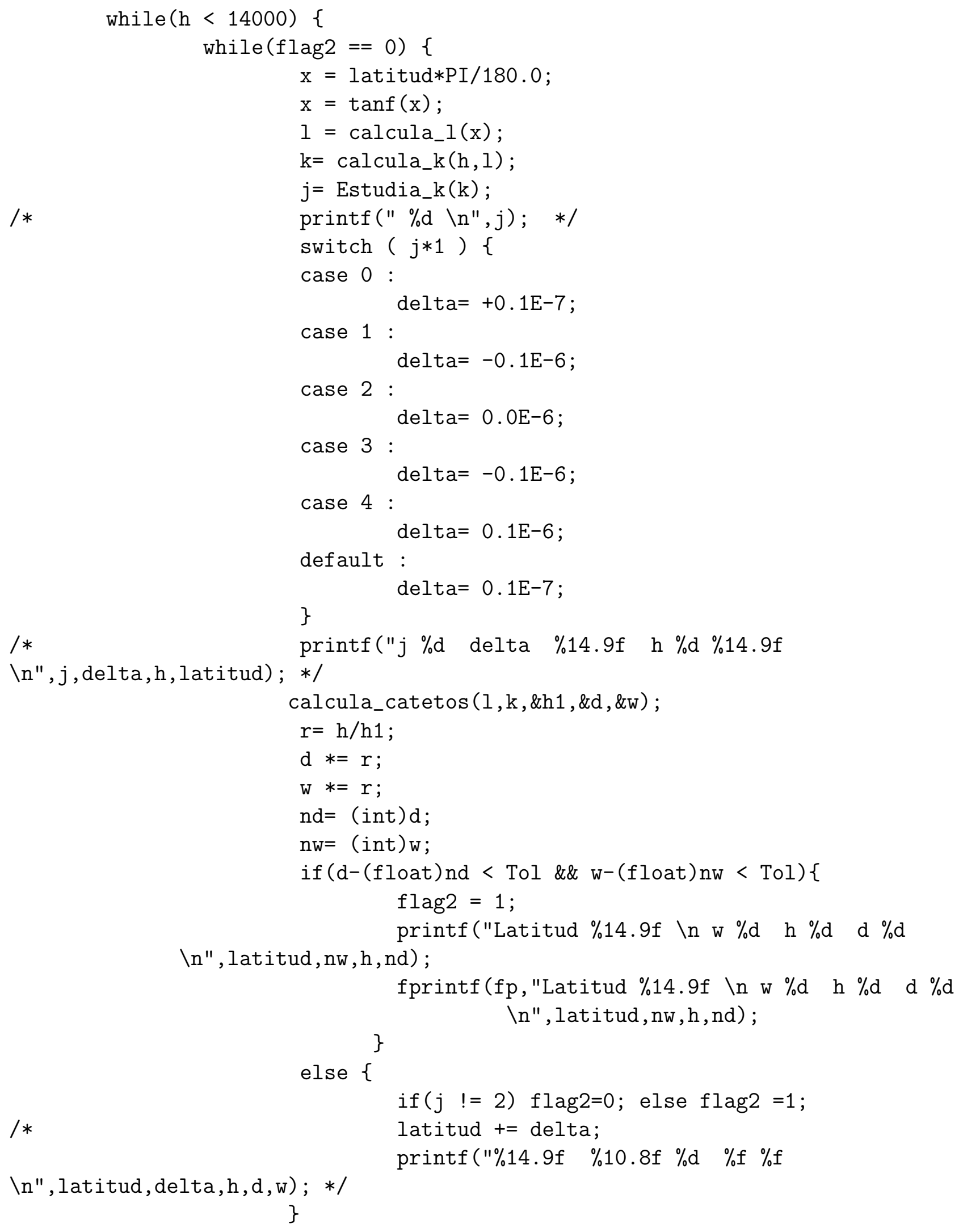




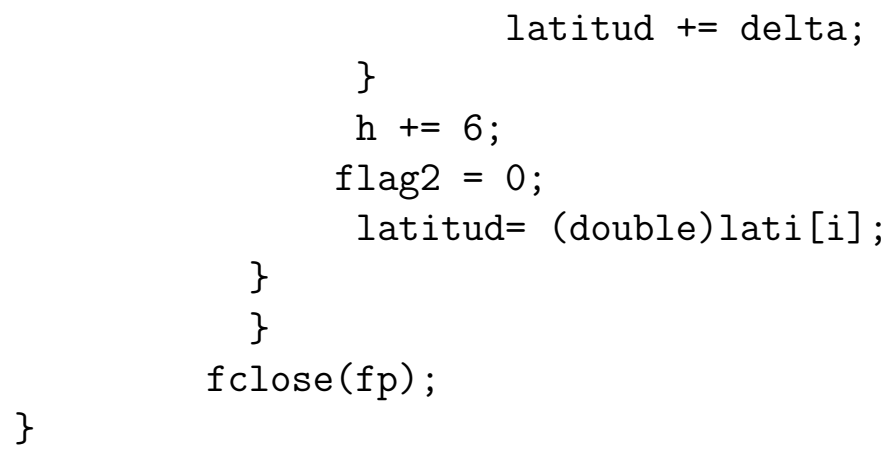

/* Fin de Programa */ 